\title{
High-Quality Genome Resource of the Pathogen of Botryosphaeria dothidea Causing Kiwifruit Soft Rot
}

\author{
Kuan Liang, ${ }^{1}$ Jianbin Lan, ${ }^{2}$ Baoquan Wang, ${ }^{3}$ Yuanyuan Liu, ${ }^{1}$ Qi Lu, ${ }^{1}$ and Pu Liu ${ }^{1, \dagger}$ \\ ${ }^{1}$ School of Horticulture, Anhui Agricultural University, Hefei 230036, People's Republic of China \\ ${ }^{2}$ College of Landscape Architecture and Life Science, Chongqing University of Arts and Sciences, \\ Chongqing 402160, People's Republic of China \\ ${ }^{3}$ College of Horticulture and Landscape Architecture, Henan Institute of Science and Technology, Xinxiang \\ 453003, People's Republic of China
}

\begin{abstract}
Kiwifruit soft rot caused by the fungal pathogen Botryosphaeria dothidea is a serious disease in kiwifruit-growing regions worldwide. In this study, we reported the high-quality genome sequence of the highly virulent $B$. dothidea strain PTZ1 using PacBio Sequel techniques. In total, 100.87 million clean reads with mean read length of 9,871 bp were obtained. De novo assembly resulted in 28 contigs with a total size of $44.45 \mathrm{Mb}$. The GC content of the genome was $54.59 \%$. Furthermore, genes related to specific virulence of the strain were identified, including 259 fungal cytochrome P450s, 550 carbohydrate-active enzymes, 860 secretory proteins, and 1,182 pathogen-host interactions related proteins. The genome is a useful resource to serve as a reference to facilitate the analysis of $B$. dothidea isolates and comparative genomic studies of the necrotroph pathogens.
\end{abstract}

Kiwifruit is cultivated and consumed worldwide because of its distinctive flavor and nutritional benefits. China is the largest kiwifruit producer in the world, with cultivation areas of more than 168,000 ha and 2,035,158 t of kiwifruit produced in 2018 (http://www. fao.org/faostat/en/). Kiwifruit soft rot, caused by the fungal pathogen Botryosphaeria dothidea, is one of the most common diseases in kiwifruit-growing regions worldwide (Wang et al. 2020). Typically, this pathogen causes rapid induction of cell death on the fruit. Later, the insides of these infected fruits become visibly watery. Finally, fruits decay severely with a sour smell.

It is well known that the strains from genus Botryosphaeria are wound pathogens (Lawrence et al. 2017; Mondello et al. 2018). Among Botryosphaeria species, B. dothidea has been reported as a pathogen of hundreds of plant species, including apple, eucalyptus, grape, kiwifruit, and peach, with characteristics of broad geographical distribution and low host specificity (Li et al. 2018; Marsberg et al. 2017). B. dothidea can infect healthy tissue of apple, olive, and pistachio as endophytes through lenticels, surface cracks, and stomata (Michailides 1991; Moral et al. 2010). Disease symptoms of plants after B. dothidea infection include cankers, dieback, blight, and fruit rots. In extreme cases, it causes the death of host plants (Slippers et al. 2017; Song et al. 2018; Wang and Zhang 2019). Genome sequencing has provided insights into the lifestyle and potential of the pathogenomics system of $B$. dothidea. Recently, the genome of $B$. dothidea and $B$. kuwatsukai, causal agents of apple ring rot, were compared and showed both species expansion of pathogenicity-related genes and variations in virulence gene content during speciation (Wang et al. 2018).

$B$. dothidea has been reported as potentially the major causal organism of kiwifruit soft rot in China (Wang et al. 2020; Zhou et al. 2015) and kiwifruit shoot blights in Greece (Thomidis and

${ }^{\dagger}$ Corresponding author: P. Liu; puliu@ahau.edu.cn

K. Liang and J. B. Lan are co-first authors.

$\boldsymbol{e}$-Xtra: One supplementary table is available online.

The author(s) declare no conflict of interest.

Accepted for publication 24 October 2020.

\section{Funding}

This project was supported by the Anhui Key Research and Development Program (1804a07020105 and 201904f06020035) and Natural Science Foundation of Anhui Province of China (2008085MC78).

\section{Keywords}

kiwifruit, Botryosphaeria dothidea, fungi, genome, high virulence, soft rot 
Table 1. General features of the genome of Botryosphaeria dothidea

\begin{tabular}{lccc} 
Feature & B. dothidea PTZ1 & B. dothidea PG45 & B. dothidea CBS 115476 \\
Total genome size $(\mathrm{Mb})$ & 44.45 & 44.3 & 43.5 \\
Number of contigs & 28 & 422 & 1,711 \\
Contig N50 $(\mathrm{kp})$ & 2,684 & 352 & 86 \\
GC\% & 54.59 & 54.60 & 54.69 \\
Number of genes & 10,415 & 15,661 & 14,998 \\
Gene average length (bp) & 1,286 & $/$ & $/$ \\
Gene length/genome (\%) & 30.13 & $/$ & $/$ \\
BUSCO & S: $98.6 \% ;$ D: $1.4 \%$ & $/$ \\
\hline
\end{tabular}

a Statistics for the B. dothidea PG45 and CBS 115476 complete genome assembly (Wang et al. 2018) are provided for comparison purposes.

b Using the databases for fungi_odb9. S = complete and single-copy BUSCOs; D = complete and duplicated BUSCOs.

Exadaktylou 2010). Fungal strain PTZ1 was isolated from diseased fruits of kiwifruit cultivar Donghong in Jinzhai, Anhui, China. Pathogenicity assessment showed that PTZ1 is a highvirulence strain on kiwifruit. Based on the internal transcribed spacer sequence, PTZ1 was identified as $B$. dothidea. Genomic DNA of $B$. dothidea PTZ1 was extracted from mycelia grown in potato dextrose broth at $25^{\circ} \mathrm{C}$ for 3 days by using the cetyl trimethyl ammonium bromide method (Weiland 1997). Genome sequencing of PTZ1 was performed using PacBio long-read and Illumina short-read sequencing technologies at the Beijing Novogene Bioinformatics Technology Co. (Beijing, China). The 350-bp paired-end library was prepared and sequenced according to the manufacturer's instructions using an Illumina NovaSeq PE150 (coverage 50x). One type of 20-kb single-molecule real-time (SMRT) bell library was constructed for sequencing using PacBio Sequel platform (coverage 224x).

In total, $1,008,686$ subreads containing $9.96 \mathrm{~Gb}$ of filtered subread bases with mean read length of 9,871 bp and a subread $N_{50}$ of 10,962 bp were generated for $B$. dothidea PTZ1. In order to detect the best assembly or assemblies, SPAdes was run at series k-mer levels $(35,47$, $59,71,83,95,107$, and 119). The completeness of the genome assembly was assessed using BUSCO version 3.0.2 (Simão et al. 2015) against fungi_odb9. The genome size was estimated to be $49.73 \mathrm{Mb}$ with $\mathrm{k}-\mathrm{mer}=15$ ( Li et al. 2008, 2010). The k-mer analysis revealed a relatively low rate of heterozygosity $(0.04 \%)$, resulting in a characteristic single peak in the k-mer frequency distribution (Vurture et al. 2017). Using SMRT Link version 5.0.1 software (Ardui et al. 2018; Reiner et al. 2018), the data were de novo assembled into 28 contigs with a total size of $44.45 \mathrm{Mb}$, with a GC content of $54.59 \%$. The length of the longest contig was $3,618,270 \mathrm{bp}$, with a $\mathrm{N}_{50}$ of $2,684,432 \mathrm{bp}$. The PacBio assembly was polished using the Illumina short reads to correct base errors. AUGUSTUS (Stanke et al. 2006), SNAP (Korf 2004), and GeneMark.hmm (Lukashin and Borodovsky 1998) were used to predict genes in the assembly. EVidenceModeler was used to combine all the predicted genes from the Program to Assemble Spliced Alignments (Haas et al. 2008). A total of 10,415 protein-coding genes were identified when using B. dothidea PG45 genome as a reference (Wang et al. 2018). The predicted genes constituted $30.13 \%$ of the assembled genome, with an average length of 1,286 bp (Table 1). rRNAs were predicted by RNAmmer (Lagesen et al. 2007). tRNA and its secondary structure were analyzed using tRNAscan-SE (Lowe and Chan 2016). sRNA, snRNA, and miRNA were predicted using BLAST software against the Rfam database (Nawrocki et al. 2015). tRNA and rRNA represented 0.0283 and $0.0060 \%$ of the genome, respectively. RepeatMasker (http:// www.repeatmasker.org/) was used to predict and annotate interspersed repetitive sequences (Smit et al. 2016). The tandem repeats were analyzed by Tandem Repeats Finder. The proportion of repeat sequences was $1.76 \%$, of which long terminal repeats, long interspersed nuclear elements, and microsatellite DNA accounted for $0.95,0.63$, and $0.19 \%$, respectively.

The functional annotation of genes was performed using BLASTp (e-value $<1 e-5$, minimal alignment length percentage $>40 \%$ ) to identify the best homologs against known databases, including Gene Ontology, Kyoto Encyclopedia of Genes and Genomes, Clusters of Orthologous Groups, Non-Redundant Protein Database, Transporter Classification Database (Saier et al. 2016), Swiss-Prot (Bairoch and Apweiler 2000), Fungal Cytochrome P450s, Pathogen Host Interactions (PHI-base) (Urban et al. 2015), and Database of Fungal Virulence Factors (Lu et al. 2012). The secretory proteins were predicted by SignalP software (version 4.1) and TMHMM (version 2.0c) (Petersen et al. 2011). Carbohydrate-active enzymes were predicted by the 
Carbohydrate-Active enZYmes database (CAZy; http://www.cazy.org) (Lombard et al. 2014). We found that the PTZ1 genome contained a large number of pathogenicity- and virulencerelated genes, including 259 P450s, 550 carbohydrate-active enzymes, 860 secretory proteins, and 1,182 pathogen-host interactions related proteins (Supplementary Table S1). The genomes of $B$. dothidea PTZ1 reported here have been deposited at GenBank under the accession numbers JABUIR000000000 (BioSample SAMN15028751).

\section{Literature Cited}

Ardui, S., Ameur, A., Vermeesch, J. R., and Hestand, M. S. 2018. Single molecule realtime (SMRT) sequencing comes of age: Applications and utilities for medical diagnostics. Nucleic Acids Res. 46:2159-2168.

Bairoch, A., and Apweiler, R. 2000. The SWISS-PROT protein sequence database and its supplement TrEMBL in 2000. Nucleic Acids Res. 28:45-48.

Haas, B. J., Salzberg, S. L., Zhu, W., Pertea, M., Allen, J. E., Orvis, J., White, O., Buell, C. R., and Wortman, J. R. 2008. Automated eukaryotic gene structure annotation using EVidenceModeler and the Program to Assemble Spliced Alignments. Genome Biol. 9:R7.

Korf, I. 2004. Gene finding in novel genomes. BMC Bioinformatics 5:59.

Lagesen, K., Hallin, P., Rødland, E. A., Staerfeldt, H. H., Rognes, T., and Ussery, D. W. 2007. RNAmmer: Consistent and rapid annotation of ribosomal RNA genes. Nucleic Acids Res. 35:3100-3108.

Lawrence, D. P., Peduto Hand, F., Gubler, W. D., and Trouillas, F. P. 2017. Botryosphaeriaceae species associated with dieback and canker disease of bay laurel in northern California with the description of Dothiorella californica sp. nov. Fungal Biol. 121:347-360.

Li, G. Q., Liu, F. F., Li, J. Q., Liu, Q. L., and Chen, S. F. 2018. Botryosphaeriaceae from Eucalyptus plantations and adjacent plants in China. Persoonia 40:63-95.

Li, R., Li, Y., Kristiansen, K., and Wang, J. 2008. SOAP: Short oligonucleotide alignment program. Bioinformatics 24:713-714.

Li, R., Zhu, H., Ruan, J., Qian, W., Fang, X., Shi, Z., Li, Y., Li, S., Shan, G., Kristiansen, K., Li, S., Yang, H., Wang, J., and Wang, J. 2010. De novo assembly of human genomes with massively parallel short read sequencing. Genome Res. 20:265-272.

Lombard, V., Golaconda Ramulu, H., Drula, E., Coutinho, P. M., and Henrissat, B. 2014. The carbohydrate-active enzymes database (CAZy) in 2013. Nucleic Acids Res. 42: D490-D495.

Lowe, T. M., and Chan, P. P. 2016. tRNAscan-SE On-line: Integrating search and context for analysis of transfer RNA genes. Nucleic Acids Res. 44:W54-W57.

Lu, T., Yao, B., and Zhang, C. 2012. DFVF: Database of fungal virulence factors. Database (Oxford) 2012:bas032.

Lukashin, A. V., and Borodovsky, M. 1998. GeneMark.hmm: New solutions for gene finding. Nucleic Acids Res. 26:1107-1115.

Marsberg, A., Kemler, M., Jami, F., Nagel, J. H., Postma-Smidt, A., Naidoo, S., Wingfield, M. J., Crous, P. W., Spatafora, J. W., Hesse, C. N., Robbertse, B., and Slippers, B. 2017. Botryosphaeria dothidea: A latent pathogen of global importance to woody plant health. Mol. Plant Pathol. 18:477-488.

Michailides, T. J. 1991. Pathogenicity, distribution, sources of inoculums, and infection courts of Botryosphaeria dothidea on pistachio. Phytopathology 81: 566-573.

Mondello, V., Songy, A., Battiston, E., Pinto, C., Coppin, C., Trotel-Aziz, P., Clément, C., Mugnai, L., and Fontaine, F. 2018. Grapevine trunk diseases: A review of fifteen years of trials for their control with chemicals and biocontrol agents. Plant Dis. 102: 1189-1217.

Moral, J., Muñoz-Díez, C., González, N., Trapero, A., and Michailides, T. J. 2010. Characterization and pathogenicity of Botryosphaeriaceae species collected from olive and other hosts in Spain and California. Phytopathology 100:1340-1351.
Nawrocki, E. P., Burge, S. W., Bateman, A., Daub, J., Eberhardt, R. Y., Eddy, S. R., Floden, E. W., Gardner, P. P., Jones, T. A., Tate, J., and Finn, R. D. 2015. Rfam 12.0: Updates to the RNA families database. Nucleic Acids Res. 43:D130-D137.

Petersen, T., Brunak, S., von Heijne, G., and Nielsen, H. 2011. SignalP 4.0: Discriminating signal peptides from transmembrane regions. Nat. Methods 8:785-786.

Reiner, J., Pisani, L., Qiao, W., Singh, R., Yang, Y., Shi, L., Khan, W. A., Sebra, R., Cohen, N., Babu, A., Edelmann, L., Jabs, E. W., and Scott, S. A. 2018. Cytogenomic identification and long-read single molecule real-time (SMRT) sequencing of a Bardet-Biedl Syndrome 9 (BBS9) deletion. NPJ Genom. Med. 3:3.

Saier, M. H., Jr., Reddy, V. S., Tsu, B. V., Ahmed, M. S., Li, C., and Moreno-Hagelsieb, G. 2016. The Transporter Classification Database (TCDB): Recent advances. Nucleic Acids Res. 44:D372-D379.

Simão, F. A., Waterhouse, R. M., Ioannidis, P., Kriventseva, E. V., and Zdobnov, E. M. 2015. BUSCO: Assessing genome assembly and annotation completeness with single-copy orthologs. Bioinformatics 31:3210-3212.

Slippers, B., Crous, P. W., Jami, F., Groenewald, J. Z., and Wingfield, M. J. 2017. Diversity in the Botryosphaeriales: Looking back, looking forward. Fungal Biol. 121: 307-321.

Smit, A. F. A., Hubley, R., and Green, P. 2016. RepeatMasker Open-4.0. 2013-2015. http://www.repeatmasker.org/.

Song, Y., Li, L., Li, C., Lu, Z., Men, X., and Chen, F. 2018. Evaluating the sensitivity and efficacy of fungicides with different modes of action against Botryosphaeria dothidea. Plant Dis. 102:1785-1793.

Stanke, M., Keller, O., Gunduz, I., Hayes, A., Waack, S., and Morgenstern, B. 2006. AUGUSTUS: Ab initio prediction of alternative transcripts. Nucleic Acids Res. 34 : W435-W439.

Thomidis, T., and Exadaktylou, E. 2010. First report of Botryosphaeria dothidea causing shoot blight of kiwifruit (Actinidia deliciosa) in Greece. Plant Dis. 94:1503.

Urban, M., Pant, R., Raghunath, A., Irvine, A. G., Pedro, H., and Hammond-Kosack, K. E. 2015. The Pathogen-Host Interactions database (PHI-base): Additions and future developments. Nucleic Acids Res. 43:D645-D655.

Vurture, G. W., Sedlazeck, F. J., Nattestad, M., Underwood, C. J., Fang, H., Gurtowski, J., and Schatz, M. C. J. B. 2017. GenomeScope: Fast reference-free genome profiling from short reads. Bioinformatics 33:2202-2204.

Wang, B., Liang, X., Gleason, M. L., Zhang, R., and Sun, G. 2018. Comparative genomics of Botryosphaeria dothidea and B. kuwatsukai, causal agents of apple ring rot, reveals both species expansion of pathogenicity-related genes and variations in virulence gene content during speciation. IMA Fungus 9:243-257.

Wang, Q. W., and Zhang, C. Q. 2019. q-LAMP assays for the detection of Botryosphaeria dothidea causing Chinese hickory canker in trunk, water, and air samples. Plant Dis. 103:3142-3149.

Wang, Y., Xiong, G., He, Z., Yan, M., Zou, M., and Jiang, J. 2020. Transcriptome analysis of Actinidia chinensis in response to Botryosphaeria dothidea infection. PLoS One 15:e0227303.

Weiland, J. J. 1997. Rapid procedure for the extraction of DNA from fungal spores and mycelia. Fungal Genet. Newslett 44:60-63.

Zhou, Y., Gong, G., Cui, Y., Zhang, D., Chang, X., Hu, R., Liu, N., and Sun, X. 2015. Identification of Botryosphaeriaceae species causing kiwifruit rot in Sichuan Province, China. Plant Dis. 99:699-708. 